\title{
Multicenter Voxel-Based Morphometry Mega-Analysis of Structural Brain Scans in Obsessive-Compulsive Disorder
}

Stella J. de Wit, M.D.

Pino Alonso, M.D., Ph.D.

Lizanne Schweren, M.Sc.

David Mataix-Cols, Ph.D.

Christine Lochner, Ph.D.

José M. Menchón, M.D., Ph.D.

Dan J. Stein, M.D., Ph.D.

Jean-Paul Fouche, M.Sc.

Carles Soriano-Mas, Ph.D.

Joao R. Sato, Ph.D.

Marcelo Q. Hoexter, M.D., Ph.D.

Damiaan Denys, M.D., Ph.D.

Takashi Nakamae, M.D., Ph.D.

Seiji Nishida, M.D., Ph.D.

Jun Soo Kwon, M.D., Ph.D.

Joon Hwan Jang, M.D., Ph.D.

Geraldo F. Busatto, M.D., Ph.D.

Narcís Cardoner, M.D., Ph.D.

Danielle C. Cath, M.D., Ph.D.
Kenji Fukui, M.D., Ph.D.

Wi Hoon Jung, Ph.D.

Sung Nyun Kim, M.D.

Euripides C. Miguel, M.D., Ph.D.

Jin Narumoto, M.D., Ph.D.

Mary L. Phillips, M.D., Ph.D.

Jesus Pujol, M.D., Ph.D.

Peter L. Remijnse, M.D., Ph.D.

Yuki Sakai, M.D., Ph.D.

Na Young Shin, M.A.

Kei Yamada, M.D., Ph.D.

Dick J. Veltman, M.D., Ph.D.

Odile A. van den Heuvel, M.D., Ph.D.

\begin{abstract}
Objective: Results from structural neuroimaging studies of obsessive-compulsive disorder (OCD) have been only partially consistent. The authors sought to assess regional gray and white matter volume differences between large samples of OCD patients and healthy comparison subjects and their relation with demographic and clinical variables.
\end{abstract}

Method: A multicenter voxel-based morphometry mega-analysis was performed on 1.5-T structural $\mathrm{T}_{1}$-weighted MRI scans derived from the International OCD Brain Imaging Consortium. Regional gray and white matter brain volumes were compared between 412 adult OCD patients and 368 healthy subjects.

Results: Relative to healthy comparison subjects, OCD patients had significantly smaller volumes of frontal gray and white matter bilaterally, including the dorsomedial prefrontal cortex, the anterior cingulate cortex, and the inferior frontal gyrus extending to the anterior insula. Patients also showed greater cerebellar gray matter volume bilaterally compared with healthy subjects. Group differences in frontal gray and white matter volume were significant after correction for multiple comparisons. Additionally, group-by-age interactions were observed in the putamen, insula, and orbitofrontal cortex (indicating relative preservation of volume in patients compared with healthy subjects with increasing age) and in the temporal cortex bilaterally (indicating a relative loss of volume in patients compared with healthy subjects with increasing age).

Conclusions: These findings partially support the prevailing fronto-striatal models of OCD and offer additional insights into the neuroanatomy of the disorder that were not apparent from previous smaller studies. The group-by-age interaction effects in orbitofrontal-striatal and (para)limbic brain regions may be the result of altered neuroplasticity associated with chronic compulsive behaviors, anxiety, or compensatory processes related to cognitive dysfunction.

(Am J Psychiatry 2014; 171:340-349)

$\mathrm{S}_{\mathrm{m}}$ basal ganglia-thalamic loops and the limbic circuit have been associated with obsessive-compulsive disorder (OCD) symptoms and associated neurocognitive dysfunctions, but probably also extend to other brain circuits (1). With voxelbased morphometry, it is possible to assess alterations in regional gray and white matter volume across the whole brain in an automated unbiased fashion.
Recent meta-analyses (2-5) that included 10-14 partially overlapping adult and pediatric voxel-based morphometry studies consistently showed smaller frontal gray matter volume in OCD, mainly of the dorsomedial prefrontal and anterior cingulate cortex (2-5), regions associated with cognitive control and performance monitoring (6). Findings in other brain regions, such as the orbitofrontal and inferior frontal cortex, which are implicated in behavioral 
inhibition and flexibility $(1,7)$, have been less consistent, with reports of normal $(2,3)$, greater $(4)$, or smaller $(4,5)$ gray matter volumes. Greater striatal volume is often found in OCD patients compared with healthy subjects (2-5), whereas reports on gray matter volume alterations outside the frontal-striatal circuit in OCD are less consistent (2-5). The involvement of white matter volume alterations in OCD remains unclear as well, with either nonsignificant findings (e.g., reference 8), smaller dorsal frontal $(9,10)$ and temporal white matter volume (11), or greater ventral frontal white matter volume (10). Although white matter volume information is automatically available in voxel-based morphometry, many studies do not report such findings, suggesting a reporting bias. Inconsistent findings can probably be attributed to differences between individual studies in the analysis methods used, heterogeneity of study samples (e.g., varying age groups), and lack of power because of limited sample sizes.

It has been suggested that regional brain volume abnormalities in OCD may result from altered neurodevelopment (endophenotype model), with additional effects of abnormal aging trajectories (8). Development- and agingdependent effects on brain structure in OCD would explain the differential effects that inclusion of pediatric samples had on previous meta-analytical results (2-4). Assessing the effects of aging on brain structure in OCD is important, since age-by-disorder interactions may provide leads as to whether changes in brain structure are related to developing the disorder (present in young patients) or are the result of persistent OCD symptoms, compensatory processes, or treatment effects (present in older patients). Additionally, heterogeneity in clinical characteristics such as OCD illness severity (2), illness onset time or duration, medication exposure (12), comorbidity $(13,14)$, and symptom dimensions $(8,9)$ may affect regional brain volume. In meta-analyses, only group-level demographic and clinical information is available, so accounting for effects of participant-level variability is problematic. An imagebased mega-analysis, in which a large sample of raw images from multiple sources is pooled and processed in a uniform preprocessing and analysis pipeline, improves statistical power compared with meta-analyses because of noise reduction and information preservation (15). Moreover, compared with previously published well-powered independent studies (e.g., reference 8), an image-based megaanalysis may have greater sensitivity in revealing significant but small effects and additionally allows generalization of findings across ethnic groups. Multicenter data pooling for voxel-based morphometry mega-analysis in other neuropsychiatric disorders has been proven successful (e.g., reference 16).

In this study, we aimed to overcome the limitations of previous structural brain imaging research in OCD by pooling data from six academic OCD centers, across three continents (Asia, Europe, and South America), participating in the international OCD Brain Imaging Consortium.
With high-quality data from 412 adult OCD patients and 368 healthy comparison subjects, this is, to our knowledge, the largest structural neuroimaging study in OCD conducted so far. This approach provides greater power to study subtle alterations in regional gray and white matter volume and to examine the effects of demographic and clinical factors, including medication use, comorbidity, and OCD symptom dimensions. We hypothesized that OCD patients would exhibit abnormalities in frontal-striatal-thalamic and (para) limbic gray matter and interconnecting white matter. Additionally, we hypothesized age-by-disorder interaction effects in relevant structures previously implicated in the disorder.

\section{Method}

\section{Participants}

Six research centers contributed data to the OCD Brain Imaging Consortium (VU University Medical Center, Amsterdam; Hospital de Bellvitge, Barcelona, Spain; Institute of Psychiatry, King's College London, London; University of São Paulo Medical School, São Paulo, Brazil; Kyoto Prefectural University of Medicine Hospital, Kyoto, Japan; and Seoul National University College of Medicine, Seoul, Republic of Korea). Each study was approved by the local ethical review board, and all participants provided written informed consent to participate in the different studies performed at each center. All centers obtained permission to participate in the consortium from their local ethical review board, and permission for multicenter data analysis was obtained from the ethical review board of the VU University Medical Center.

The study included 436 OCD patients and 382 healthy comparison subjects who underwent 1.5 - $\mathrm{T}$ structural $\mathrm{T}_{1}$-weighted MRI scanning (see Tables 1 and 2). All participants were screened for DSM-IV axis I disorders with a standardized structured interview (see the Supplemental Methods section in the data supplement that accompanies the online edition of this article). Patients were recruited through local outpatient or specialist OCD clinics, and healthy subjects through local advertisements. Exclusion criteria were age under 18 or over 65 years, a current psychotic disorder, a recent history of a substance use disorder, mental retardation, and severe organic or neurological pathology, except tic disorder. In patients, psychiatric comorbidity (including tic disorders) was not an exclusion criterion provided that OCD was the primary diagnosis. Healthy comparison subjects had no current axis I psychiatric disorders.

Detailed sociodemographic and clinical data were collected at each center. Age at onset, OCD illness severity ratings, symptom dimension scores, and current medication use were available from most patients (see the online data supplement).

\section{Data Acquisition and Quality Control}

The parameters of the structural scans are listed in Table S1 in the online data supplement. After extensive quality checking (see the data supplement and Table 2), 780 participants (412 patients and 368 healthy subjects; Table 1) were included in the analysis. Data on 530 of these 780 participants have not been published before or been included in previous meta-analyses (see Table S1 in the data supplement).

\section{Statistical Analysis}

Group differences in sample characteristics were assessed with SPSS, version 20 (IBM, Armonk, N.Y.). The significance threshold was set at 0.05 , two-tailed. 
TABLE 1. Demographic and Clinical Characteristics of OCD Patients and Healthy Comparison Subjects

\begin{tabular}{|c|c|c|c|c|c|c|c|}
\hline \multirow[t]{2}{*}{ Characteristic $^{\mathrm{a}}$} & \multicolumn{2}{|c|}{ OCD Patients $(\mathrm{N}=412)$} & \multicolumn{2}{|c|}{ Healthy Subjects $(\mathrm{N}=368)$} & \multicolumn{3}{|c|}{ Statistical Analysis } \\
\hline & Mean & SD & Mean & SD & $\mathrm{t}$ & df & $\mathrm{p}$ \\
\hline Age (years) & 32.1 & 9.6 & 30.2 & 9.3 & 2.9 & 778 & 0.004 \\
\hline Education level (years) & 13.7 & 2.8 & 14.6 & 3.1 & -4.0 & 760 & $<0.001$ \\
\hline OCD illness severity score ${ }^{b}$ & 24.9 & 6.2 & & & & & \\
\hline Age at onset of clinical symptoms (years) ${ }^{C}$ & 20.1 & 8.7 & & & & & \\
\hline Total gray matter volume $(\mathrm{mL})$ & 700.6 & 63.3 & 705.1 & 66.2 & -0.9 & 778 & 0.33 \\
\hline \multirow[t]{2}{*}{ Total white matter volume $(\mathrm{mL})$} & 513.6 & 49.2 & 511.7 & 52.6 & 0.5 & 778 & 0.60 \\
\hline & $\mathrm{N}$ & $\%$ & $\mathrm{~N}$ & $\%$ & $x^{2}$ & & $\mathrm{p}$ \\
\hline Male & 202 & 49.0 & 195 & 53.0 & 1.2 & 1 & 0.28 \\
\hline Right-handed & 354 & 85.9 & 330 & 89.7 & 1.0 & 2 & 0.65 \\
\hline Ethnicity & & & & & 2.7 & 2 & 0.26 \\
\hline Caucasian & 195 & 47.3 & 192 & 52.2 & & & \\
\hline Asian & 171 & 41.5 & 146 & 39.7 & & & \\
\hline Other & 6 & 1.5 & 11 & 3.0 & & & \\
\hline Medication use at time of scan & 176 & 42.7 & 0 & 0.0 & 210.1 & 1 & $<0.001$ \\
\hline Current comorbidity & 149 & 36.2 & 0 & 0.0 & 174.0 & 1 & $<0.001$ \\
\hline Lifetime comorbidity & 213 & 51.7 & 7 & 1.9 & 253.7 & 1 & $<0.001$ \\
\hline Prepubertal OCD onset & 51 & 13.0 & & & & & \\
\hline \multicolumn{8}{|l|}{ OCD symptom dimensions ${ }^{c}$} \\
\hline Aggressive/checking & 236 & 57.2 & & & & & \\
\hline Contamination/cleaning & 202 & 49.0 & & & & & \\
\hline Symmetry/ordering & 168 & 40.8 & & & & & \\
\hline Sexual/religious & 130 & 31.6 & & & & & \\
\hline Hoarding & 87 & 21.1 & & & & & \\
\hline
\end{tabular}

${ }^{a}$ Missing data for characteristics ranged from $\mathrm{N}=0$ to $\mathrm{N}=40$.

${ }^{b}$ As measured with the Yale-Brown Obsessive Compulsive Scale (YBOCS) total score. Mean score on the obsessions subscale, 12.6 (SD=3.4); mean score on the compulsions subscale, $12.3(\mathrm{SD}=3.8)$.

${ }^{c}$ As measured with the YBOCS symptom checklist.

TABLE 2. Number of Scans Provided and Included for OCD Patients and Healthy Comparison Subjects at Each Center

\begin{tabular}{|c|c|c|c|c|c|c|c|c|c|}
\hline \multirow[b]{2}{*}{ Site } & \multicolumn{3}{|c|}{ Initial Number of Scans } & \multicolumn{3}{|c|}{ Included After Quality Check } & \multicolumn{3}{|c|}{ Exclusions } \\
\hline & OCD Patients & Healthy Subjects & Total & OCD Patients & Healthy Subjects & Total & Pathology & Poor Quality ${ }^{\mathrm{a}}$ & Total \\
\hline Amsterdam & 55 & 50 & 105 & 53 & 49 & 102 & 1 & 2 & 3 \\
\hline Barcelona & 88 & 103 & 191 & 86 & 102 & 188 & 1 & 2 & 3 \\
\hline Kyoto & 89 & 48 & 137 & 84 & 48 & 132 & 1 & 4 & 5 \\
\hline London & 55 & 37 & 92 & 44 & 33 & 77 & 3 & 12 & 15 \\
\hline São Paulo & 60 & 46 & 106 & 58 & 39 & 97 & 0 & 9 & 9 \\
\hline Seoul & 89 & 98 & 187 & 87 & 97 & 184 & 1 & 2 & 3 \\
\hline Total & 436 & 382 & 818 & 412 & 368 & 780 & 7 & 31 & 38 \\
\hline
\end{tabular}

a Poor quality is an umbrella term referring to scans being either nonsegmentable or having artifacts related to motion, poor contrast, or imaging.

Imaging data processing and analysis were done using optimized voxel-based morphometry (17) in the SPM8 program (www.fil.ion.ucl.ac.uk/spm/software/spm8). After manual reorientation, images were segmented into gray and white matter with the "New Segment" tool (default settings) and resampled to a resolution of $1.5 \times 1.5 \times 1.5 \mathrm{~mm}$. Using the diffeomorphic anatomical registration through exponential Lie algebra algorithm (DARTEL [17], default settings), an analysis-specific group template was created (i.e., with $\mathrm{N}=780$ for the main group analysis and $\mathrm{N}=412$ for within-patient analyses). The gray and white matter images were first warped to the group-specific template using the flow fields (specifying the deformations between each participant's individual scan and the group-specific template) and subsequently to Montreal Neurological Institute (MNI) standard space using the warping parameters of the group template to the tissue prior map in standard space. To preserve regional volumetric information, images were modulated during the warping. To increase the signal-to-noise ratio, images were smoothed with a $10-\mathrm{mm}$ isotropic Gaussian kernel.

Group effects and age-by-group interactions on regional gray and white matter volume were investigated by feeding the preprocessed gray and white matter images of OCD patients $(\mathrm{N}=412)$ and healthy subjects $(\mathrm{N}=368)$ into general linear models that always included total gray or white matter, scan sequence, age, gender, and education level as covariates. Aging in healthy individuals is associated with both bilinear (an even rate of change over time) and nonlinear (quadratic; acceleration/deceleration of the rate of change over time) patterns of regional brain volume loss or preservation as compared with global brain volume loss (see reference 18 , for example). We therefore assessed the effect of age and age- 
FIGURE 1. Regional Gray and White Matter Volume Differences Between OCD Patients ( $=412)$ and Healthy Comparison Subjects $(\mathrm{N}=368)^{\mathrm{a}}$

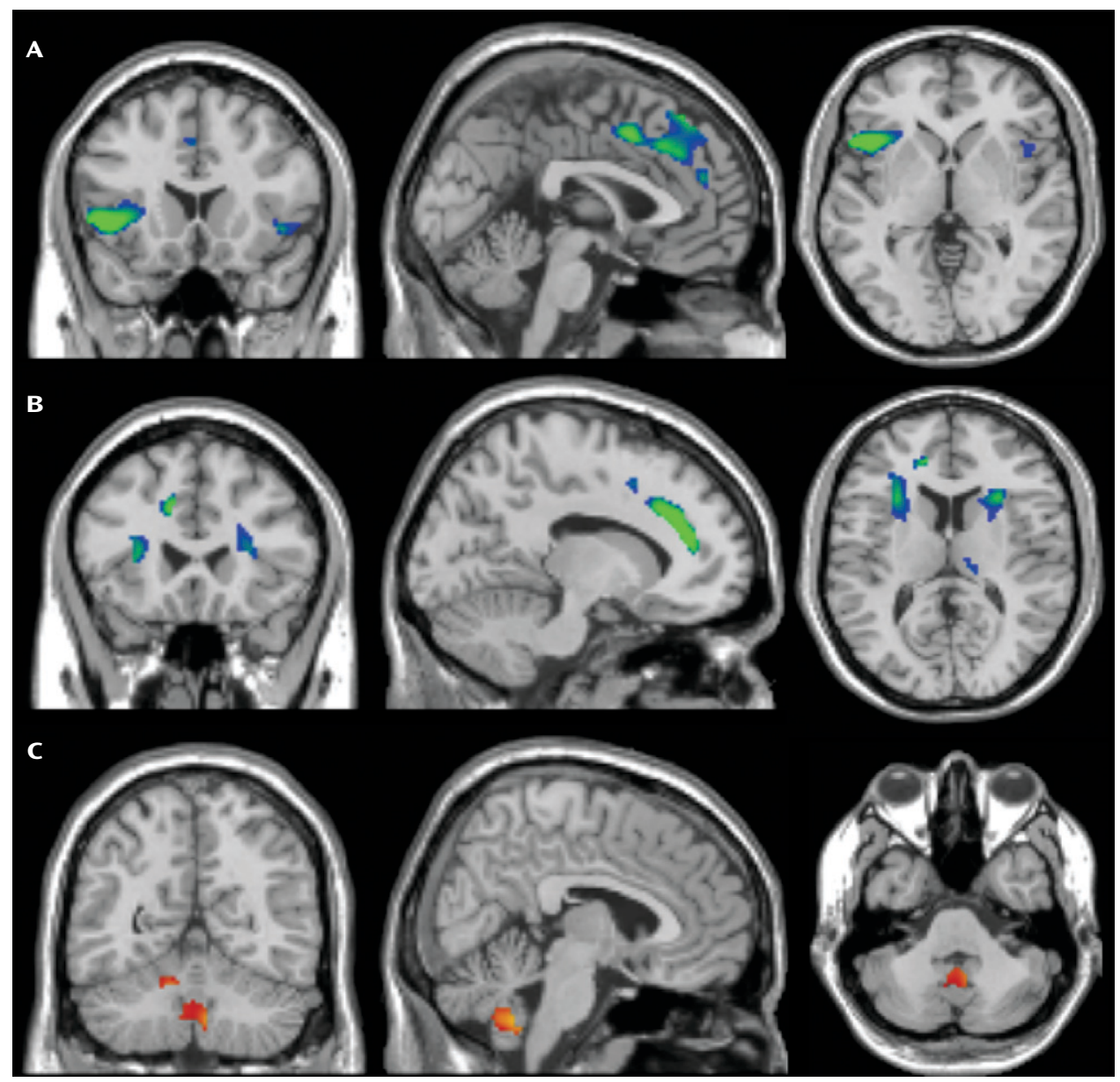

a Compared with healthy subjects, OCD patients exhibit smaller (blue/green) regional medial and inferior frontal gray (panel A) and white (panel B) matter volume and greater (red/yellow) regional cerebellar gray matter volume (panel C). Brain slices in panels A-C are in coronal (left), sagittal (middle), and axial (right) direction; left side of brain is on the left. MNI coordinates ( $x, y, z)$ of panel A, $-1,18,0$; of panel B, -11 , 23, 12; of panel C, $6,-55,-40$. All presented imaging results are T-statistic images overlain over the SPM8 single-subject $\mathrm{T}_{1}$ standard brain with MRIcron (www.mccauslandcenter.sc.edu/mricro/mricron) and thresholded at $p<0.001$ uncorrected with a minimum cluster extent of 100 voxels. $T$ values are displayed from red/blue $(T=3.1)$ to yellow/green $(T \geq 4.0)$.

by-group interactions using both age (bilinear) and age squared (nonlinear) as covariates (18). Multiple linear regression models were used to investigate the effects of age at onset, illness duration, severity of OCD, comorbid major depressive disorder, comorbid anxiety disorders, OCD symptom dimensions, and medication use within patients. To maximize statistical sensitivity by including only relevant within-brain voxels, absolute masking was applied at an estimated optimal threshold of 0.2 (19). For maximal comparability with previous results in the field (e.g., references 9, 20, 21; see also references 2-5), resulting T-maps were thresholded at $\mathrm{p}<0.001$ uncorrected at the voxel level and a minimum cluster extent of 100. Additionally, whole-brain family-wise error, multiple comparison, and nonstationarity corrected cluster-level statistics are reported at a family-wise error corrected p threshold of 0.05 (22).

\section{Results}

\section{Group Comparisons}

The OCD group $(\mathrm{N}=412)$ and the healthy comparison group $(\mathrm{N}=368)$ did not differ in gender, handedness, ethnicity, and total gray matter and white matter volume, but on average the OCD group was significantly older, had a lower education level, and scored higher on all clinical measures (Table 1). As shown in Figure 1, compared with healthy subjects, patients had smaller regional gray matter volume in the left and right dorsomedial frontal cortex (Brodmann's area [BA] 6/8/9) extending to the anterior cingulate cortex (BA 24/32) and inferior frontal cortex/ anterior insula (BA 13/47); greater cerebellar gray matter volume bilaterally; and smaller frontal white matter volume bilaterally (Table 3). Post hoc analyses (see the Supplemental Results section in the online data supplement) showed that scan sequence/site and current medication use did not affect group interaction results.

To ensure that the observed group differences were not confounded by age or education level, we performed a post hoc analysis $(\mathrm{N}=645)$ in patient $(\mathrm{N}=329)$ and healthy $(\mathrm{N}=316)$ samples that were matched on all demographic 
TABLE 3. Regional Brain Volume Differences Between OCD Patients $(N=412)$ and Healthy Comparison Subjects $(N=368)^{a}$

\begin{tabular}{|c|c|c|c|c|c|c|c|c|}
\hline \multirow[b]{2}{*}{ Region and Contrast } & \multirow[b]{2}{*}{ Side } & \multirow[b]{2}{*}{$\mathrm{BA}$} & \multirow[b]{2}{*}{$\mathrm{k}_{\mathrm{e}}$} & \multicolumn{3}{|c|}{ MNI Coordinates } & \multirow[b]{2}{*}{ Z } & \multirow[b]{2}{*}{$p^{b}$} \\
\hline & & & & $x$ & y & z & & \\
\hline \multicolumn{9}{|l|}{ Gray matter } \\
\hline \multicolumn{9}{|l|}{ Healthy subjects $>$ OCD patients } \\
\hline Inferior frontal cortex/anterior insula & $\mathrm{L}$ & $47 / 13 / 45$ & 1,295 & -44 & 17 & -3 & 4.97 & 0.05 \\
\hline \multirow[t]{2}{*}{$\begin{array}{l}\text { Dorsomedial prefrontal cortex/anterior } \\
\text { cingulate cortex }\end{array}$} & $R / L$ & $32 / 9 / 8 / 6 / 24$ & 2,008 & $\begin{array}{r}0 \\
-3\end{array}$ & $\begin{array}{r}9 \\
38\end{array}$ & $\begin{array}{l}45 \\
36\end{array}$ & $\begin{array}{l}4.11 \\
3.95\end{array}$ & 0.01 \\
\hline & & & & 2 & 41 & 51 & 3.91 & \\
\hline Inferior frontal cortex/anterior insula & $\mathrm{R}$ & $47 / 13$ & 165 & 47 & 17 & -5 & 3.77 & 0.33 \\
\hline \multicolumn{9}{|l|}{ OCD patients $>$ healthy subjects } \\
\hline \multirow[t]{2}{*}{$\begin{array}{l}\text { Cerebellum (extending through } \\
\text { flocculonodular, anterior, and } \\
\text { posterior lobes) }\end{array}$} & $R / L$ & & 581 & $\begin{array}{r}9 \\
-5 \\
-5\end{array}$ & $\begin{array}{l}-48 \\
-51 \\
-57\end{array}$ & $\begin{array}{l}-48 \\
-44 \\
-36\end{array}$ & $\begin{array}{l}3.96 \\
3.48 \\
3.24\end{array}$ & 0.74 \\
\hline & $\mathrm{L}$ & & 223 & -12 & -56 & -26 & 3.51 & 0.81 \\
\hline \multicolumn{9}{|l|}{ White matter } \\
\hline \multicolumn{9}{|l|}{$\begin{array}{l}\text { Healthy subjects }>\text { OCD patients } \\
\text { Frontal white matter }\end{array}$} \\
\hline Medial & $\mathrm{L}$ & & 945 & $\begin{array}{l}-11 \\
-12\end{array}$ & $\begin{array}{l}33 \\
39\end{array}$ & $\begin{array}{l}27 \\
18\end{array}$ & $\begin{array}{l}4.71 \\
4.70\end{array}$ & 0.04 \\
\hline Inferior/middle & $\mathrm{L}$ & & 968 & $\begin{array}{l}-30 \\
-29\end{array}$ & $\begin{array}{l}29 \\
20\end{array}$ & $\begin{array}{r}6 \\
12\end{array}$ & $\begin{array}{l}3.91 \\
3.78\end{array}$ & 0.07 \\
\hline Medial & L & & 104 & -9 & 12 & 47 & 3.52 & 0.52 \\
\hline \multirow[t]{3}{*}{ Inferior/middle } & $\mathrm{R}$ & & 592 & 29 & 20 & 12 & 3.82 & 0.23 \\
\hline & & & & 24 & 27 & 23 & 3.30 & \\
\hline & & & & 35 & 27 & 6 & 3.12 & \\
\hline \multirow[t]{2}{*}{ Thalamic region } & $\mathrm{R}$ & & 216 & 15 & -26 & 9 & 3.44 & 0.50 \\
\hline & & & & 11 & -20 & 14 & 3.29 & \\
\hline
\end{tabular}

\footnotetext{
${ }^{a}$ Analysis of covariance thresholded at $p<0.001$ uncorrected and a minimum cluster extent $\left(k_{e}\right)$ of 100 voxels. Table shows local maxima more than $8.0 \mathrm{~mm}$ apart. Results are corrected for age, gender, education level, total gray matter or white matter volume, and scan sequence. $\mathrm{BA}=$ Brodmann's area; $\mathrm{MNI}=$ Montreal Neurological Institute; $\mathrm{L}=$ left; $\mathrm{R}=$ right; $\mathrm{ns}=$ not significant.

${ }^{b}$ Whole-brain cluster family-wise error corrected and nonstationarity corrected $\mathrm{p}$ value.
}

variables (see the data supplement). Results of this post hoc analysis were similar to those in the larger sample (see Table S2 in the data supplement).

\section{Group-by-Age Interactions}

The linear group-by-age interaction analyses (Table 4, Figure 2) showed a relative preservation of right putamen/ insular gray matter volume with aging in OCD patients compared with healthy subjects and greater loss of temporal gray matter volume bilaterally. The nonlinear analysis showed a greater loss of fusiform and parahippocampal gray matter and preservation of frontal white matter volume with aging in OCD patients compared with healthy subjects (see the data supplement for within-group effects).

Post hoc matched samples analysis (see Table S3 in the data supplement) confirmed these group-by-age interaction effects, with the putamen/insula cluster reaching family-wise error corrected significance and the nucleus accumbens and inferior and orbitofrontal gray matter reaching the statistical threshold.

\section{Effect of Clinical Variables on Brain Structure in Patients}

Illness severity, illness duration, and age at onset were not significantly related to regional brain volume. Patients on medication at the time of the scan $(\mathrm{N}=176$; mean age, 33.2 years; median time on medication, 24 weeks; missing, $\mathrm{N}=46$ ) showed significantly greater operculum/posterior insula gray matter volumes and smaller middle frontal gray matter and posterior frontal white matter volumes compared with patients who were not on medication $(\mathrm{N}=222$; mean age, 30.9 years; see Table $\mathrm{S} 4$ in the data supplement). Since medicated and unmedicated patients differed in several characteristics, we used stepwise multiple linear regression (see the data supplement) to assess whether the results truly could be attributed to medication. After controlling for demographic and clinical variability, medication effects remained significant only in middle frontal gray matter volume $(\mathrm{p}=0.014)$.

OCD patients with one or more comorbid anxiety disorders ( $\mathrm{N}=83)$ had greater left cerebellar gray matter volume and smaller volumes of the right temporo-occipital gray matter, left superior frontal gyrus, midcingulum, and insula compared with those without comorbid anxiety $(\mathrm{N}=190$; missing data, $\mathrm{N}=139$; for a detailed description of analyses, see the Supplemental Results section and Table S5 in the data supplement). Patients with a lifetime diagnosis of depression $(\mathrm{N}=101)$ had a smaller right supplementary motor area gray matter volume compared with patients without $(\mathrm{N}=287$; 
TABLE 4. Group-by-Age Interactions on Regional Brain Volume in OCD Patients $(\mathrm{N}=412)$ and Healthy Comparison Subjects $(\mathrm{N}=368)^{\mathrm{a}}$

\begin{tabular}{|c|c|c|c|c|c|c|c|c|}
\hline \multirow[b]{2}{*}{ Region and Interaction } & \multirow[b]{2}{*}{ Side } & \multirow[b]{2}{*}{ BA } & \multirow[b]{2}{*}{$\mathrm{k}_{\mathrm{e}}$} & \multicolumn{3}{|c|}{ MNI Coordinates } & \multirow[b]{2}{*}{ Z } & \multirow[b]{2}{*}{$p^{b}$} \\
\hline & & & & $x$ & $\mathrm{y}$ & z & & \\
\hline \multicolumn{9}{|l|}{ Gray matter } \\
\hline \multicolumn{9}{|l|}{$\begin{array}{l}\text { Relative preservation with aging in OCD patients } \\
\text { compared with healthy subjects }\end{array}$} \\
\hline \multicolumn{9}{|l|}{ Linear } \\
\hline \multirow[t]{2}{*}{ Putamen extending to insula } & $\mathrm{R}$ & 13/NA & 585 & 42 & 6 & -6 & 3.67 & 0.27 \\
\hline & & & & 33 & 5 & -9 & 3.60 & \\
\hline \multicolumn{9}{|l|}{ Nonlinear: ns } \\
\hline \multicolumn{9}{|l|}{$\begin{array}{l}\text { Relative loss with aging in OCD patients compared } \\
\text { with healthy subjects }\end{array}$} \\
\hline \multicolumn{9}{|l|}{ Linear } \\
\hline Middle temporal gyrus & $\mathrm{R}$ & 21 & 147 & 56 & -35 & -8 & 3.94 & 0.37 \\
\hline Inferior temporal gyrus & $\mathrm{L}$ & 20 & 245 & -60 & -39 & -23 & 3.91 & 0.55 \\
\hline \multicolumn{9}{|l|}{ Nonlinear } \\
\hline \multirow[t]{2}{*}{ Fusiform gyrus } & L & $37 / 19$ & 546 & -47 & -62 & -21 & 4.39 & 0.16 \\
\hline & & & & -38 & -54 & -12 & 3.25 & \\
\hline \multirow[t]{2}{*}{ Parahippocampal gyrus extending to thalamus } & L & $35 / 27$ & 244 & -20 & -33 & 4 & 3.76 & 0.45 \\
\hline & & & & -20 & -30 & -20 & 3.21 & \\
\hline \multicolumn{9}{|l|}{ White matter } \\
\hline \multicolumn{9}{|l|}{$\begin{array}{l}\text { Relative preservation with aging in OCD patients } \\
\text { compared with healthy subjects }\end{array}$} \\
\hline \multicolumn{9}{|l|}{ Linear: ns } \\
\hline \multicolumn{9}{|l|}{ Nonlinear } \\
\hline \multicolumn{9}{|l|}{ Frontal white matter } \\
\hline Middle & L & & 447 & -18 & 48 & 4 & 4.35 & 0.17 \\
\hline Inferior & $\mathrm{L}$ & & 135 & -33 & 30 & 0 & 3.80 & 0.51 \\
\hline Medial & $\mathrm{R}$ & & 189 & 20 & 38 & 0 & 3.28 & 0.56 \\
\hline \multicolumn{9}{|l|}{$\begin{array}{l}\text { Relative loss with aging in OCD patients compared } \\
\text { with healthy subjects }\end{array}$} \\
\hline Linear, nonlinear: ns & & & & & & & & \\
\hline
\end{tabular}

missing data, $\mathrm{N}=24)$. Patients with current comorbid major depressive disorder $(\mathrm{N}=46)$ had smaller left frontal gray and white matter volumes compared with those without $(\mathrm{N}=287)$.

OCD symptom dimension analyses in patients $(\mathrm{N}=331$; missing data, $\mathrm{N}=81$ ) showed an association of the presence of aggression/checking symptoms with greater lingual gyrus volume and smaller superior parietal gray and white matter volumes, of hoarding with a smaller right cerebellar gray matter volume, of sexual/religious obsessions with greater middle temporal volume, and of symmetry/ordering with a smaller fusiform gray matter volume (see the Supplemental Results section and Table S4 in the data supplement).

\section{Discussion}

To our knowledge, this is the largest neuroimaging study in OCD conducted to date. The large sample size allowed sufficient statistical power to detect possible subtle abnormalities in OCD and to examine potential effects of age and clinical characteristics on brain volumes.

\section{Frontal and Cerebellar Volume Changes in OCD}

In agreement with prevailing frontal-striatal models of OCD pathophysiology, we found smaller volumes of the left and right medial and inferior frontal gray matter and adjacent white matter in OCD patients compared with healthy subjects. Smaller medial frontal gray matter volume in OCD is in accordance with previous voxel-based morphometry studies (2-5) and with studies showing reduced levels of $N$-acetylaspartate, a measure of neuronal density, in this region in OCD (23). Smaller volumes of the left and right inferior frontal gyrus extending to the anterior insula were reported once before in OCD (21) but are in contrast with other studies showing greater (e.g., reference 4 ) or normal $(5,8)$ inferior frontal cortex/anterior insula volume. Our finding of smaller medial and inferior frontal white matter volumes bilaterally in OCD is notable considering inconsistent previous reports (e.g., references 8-10) and converges with studies showing altered frontal-striatal white matter microstructure in OCD (5). Frontal volume changes may be related to impairments in cognitive functions that have been 
FIGURE 2. Group-by-Age Interactions in the Putamen and Orbitofrontal Cortex in OCD Patients $(\mathrm{N}=412)$ and Healthy Comparison Subjects $(\mathrm{N}=368)^{\mathrm{a}}$
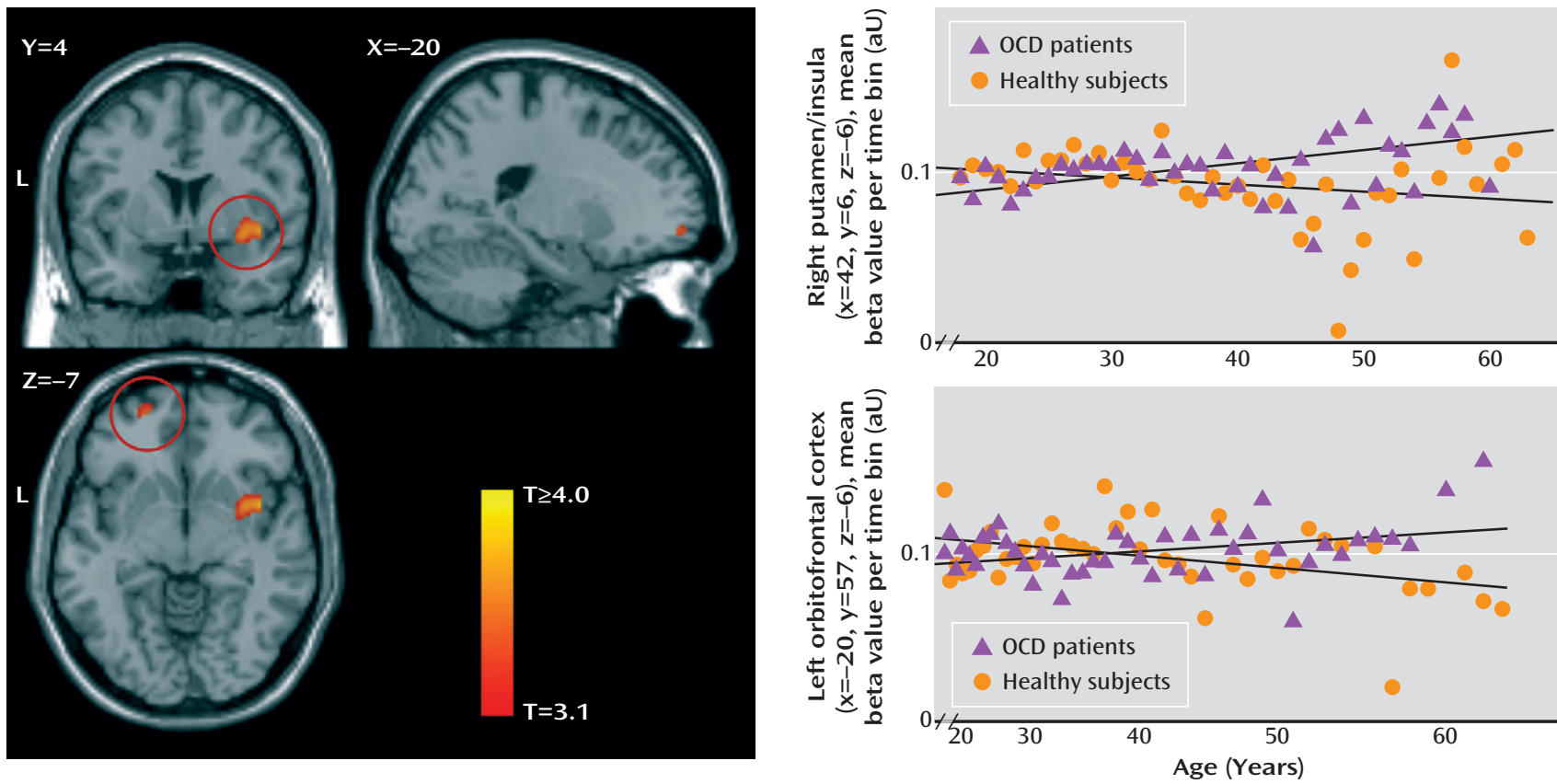

${ }^{a}$ The left-hand panel shows relative preservation of putamen/insular (circle in upper image) and orbitofrontal (circle in lower image) gray matter volume with increasing age in OCD patients compared with healthy subjects. T-statistic images are from linear and nonlinear age-bygroup analyses and are thresholded at $p<0.001$ uncorrected with a minimum cluster extent of 98 voxels for illustration purposes. In the righthand panel, the line plots show mean peak voxel values of the right putamen/insula cluster (upper plot; linear age scale) and left orbitofrontal frontal cortex (lower plot; quadratic age scale) in patients and healthy subjects. Displayed values are the mean parameter estimate (which relates to relative gray matter volume) per time bin (1 year) per group in arbitrary units (aU). L=left.

consistently demonstrated in OCD patients, such as cognitive control and emotion regulation (6), and are associated with altered activity in the dorsomedial prefrontal cortex and inferior frontal cortex/anterior insula $(1,7)$. Smaller medial frontal gray matter volume has been reported in anxiety and depression $(3,24)$, and in our sample it was more pronounced in patients with additional comorbid anxiety and/ or depression, which may thus be indicative of a common pathophysiological mechanism across affective disorders related to a shared deficit in emotion regulation $(3,24)$. On the other hand, smaller inferior frontal cortex/anterior insula volumes may be a neuroanatomical characteristic more specific to OCD and related disorders $(14,24,25)$, although this speculation awaits empirical confirmation.

We found greater cerebellar gray matter volume in OCD patients relative to healthy subjects. The cerebellum is structurally and functionally connected to the parallel cortico-striatal-thalamic-cortical circuits and is thought to integrate cortico-striatal information flow (26). There is accumulating evidence that the cerebellum, besides its role in motor control, is also involved in cognitive and emotional regulatory processes $(13,26,27)$. Smaller cerebellar volume has been found in disorders often comorbid with OCD, such as Tourette's disorder (13), trichotillomania (28), depression, and anxiety (29). Previous studies in adult OCD populations showed smaller (20), normal (2), or greater $(4,5,8)$ cerebellar gray matter volume. The cerebellar volume alterations we observed are more extensive than those reported in previous studies $(4,5,8)$. Aberrant cerebellar activity has been shown in OCD patients during rest and task performance (1). Cerebellar volume changes may thus be directly related to cognitive dysfunction and OCD symptomatology $(26,27)$. In the context of the existing literature, our data suggest a greater role for the cerebellum in OCD illness models than is currently recognized.

\section{Altered Brain Volume Changes in OCD During Aging}

It has been suggested that abnormal regional brain volume in OCD might result from a combination of abnormal neurodevelopmental processes and additional altered aging-related trajectories due to several factors operating on multiple levels-for example, genetic variations influencing neuronal pruning and aging processes, activity-dependent neuronal plasticity associated with chronic symptoms or compensatory processes, and neurochemical effects of pharmacological treatment or stress $(30,31)$. Patients showed relative preservation of the putamen, nucleus accumbens, and inferior and orbitofrontal cortex during aging, which was in contrast to the loss associated with healthy aging (18). Evidence from electrophysiology and neuroimaging (1) studies has long indicated the involvement of the basal ganglia in OCD. Greater putamen and caudate volume is often (2-5) but not consistently found, and findings may be dependent on the age of the 
recruited samples, treatment history, or treatment status (12). We did not find striatal volume alterations in the overall OCD patient group, but in line with a previous report (8), we observed a relatively preserved volume of the putamen and nucleus accumbens during aging in patients. Given the involvement of the putamen in motor behavior, aging-related putamen volume preservation in OCD may be related to activation-induced neuroplasticity as a result of chronic compulsive behaviors (30). Ageby-group interactions in the orbitofrontal cortex and striatum, core nodes of the neural reward circuit (32), may also be the result of compensatory processes. Neuroimaging studies have shown dysfunction in this circuit in OCD during rest (33) and task performance (1), associated with impaired feedback learning, behavioral inflexibility, and a motivational bias toward negative stimuli. Additionally, parts of the inferior frontal lobe adjacent to the inferior frontal cortex/anterior insula regions showing smaller volume overall showed a relative preservation with aging in OCD patients. Besides having a role in cognitive control (6), the inferior frontal cortex/anterior insula is implicated in attention (34), interoceptive awareness (35), and disgust perception (36). Given that these functions are thought to be abnormal in OCD $(1,37)$, it may be that the observed aging effect in the inferior frontal cortex/anterior insula is related to (compensatory) activation-induced neuroplasticity.

In line with most previous reports (e.g., reference 5), we did not observe volume differences in the amygdalahippocampal complex in OCD. Our data do indicate, however, that (para)limbic parts of the medial and lateral temporal cortex, regions that are relatively preserved in healthy aging (38), show greater aging-related volume loss in OCD. The fusiform, parahippocampal, and inferior temporal cortices are involved in higher-level visual processing, visual emotional perception (39), and emotional memory formation (40). Greater volume loss in these regions may thus be related to chronic stress and the exaggerated emotional responsiveness seen in OCD $(1,31)$.

\section{Association With Clinical Characteristics}

In patients, illness severity, age at onset, and illness duration were not significantly related to regional brain volume, which is in accordance with some (e.g., references 8 , 10), but not other (e.g., reference 21) previous studies. It must be noted that age at onset was ascertained retrospectively, and therefore the negative findings must be interpreted in light of this limitation. Although topologically not converging with previous findings (e.g., references 8, 9), the results of the symptom dimension analyses broadly agree with the notion that the various subphenotypes of OCD have overlapping but also unique neural correlates (41), which may in turn reflect genetic and especially environmental influences on each of these phenotypes (42).

Consistent with previous meta-analyses $(2,3)$, the results from the main group comparisons were robust in controlling for current medication use. The within-patient medication analyses showed opposing effects of medication on dorsal and ventral frontal gray matter. Moreover, only the middle frontal gray matter finding (a region not overlapping with the main group effects) remained significant after we controlled for the differing sample characteristics of the medicated versus unmedicated patients in a multiple regression analysis. Therefore, it is possible that gray matter alterations in the middle frontal cortex may be partially attributable to current medication use, a finding that deserves further investigation, ideally using longitudinal designs (12).

\section{Limitations and Future Directions}

With our study design, we gained statistical power and the ability to assess and control for the effect of participantlevel variability by performing an image-based megaanalysis on pooled data. This experimental gain was potentially offset by the introduction of possible confounding effects, such as ethnicity, MRI scanner hardware/ sequence, and medication use-confounders that individual well-powered studies may not have. However, we think it is unlikely that these possible confounders affected our results. First, we corrected for site/scanning sequence in all the analyses. Second, group-by-site interaction effects did not explain the group results, and current medication use did not affect the main results. Furthermore, since we included age and education level as covariates in all analyses, and since our results were robust against appropriate age and education level matching with a corresponding sample size reduction, we think it safe to conclude that the reported brain volume alterations in OCD are not explained by demographic variability. A limitation of analyzing age-by-disorder interactions with a cross-sectional instead of a longitudinal study design is that aging-related intrasubject changes cannot be disentangled from possible intersubject differences (18). Assessing group and group-byage interactions in a large cohort increases statistical sensitivity, but it also introduces intersubject variation that may have caused real effects to be underestimated. Analysis of this data set with a toolbox with putative superior subcortical image segmentation (e.g., FreeSurfer) is a logical future step, since this additionally would ascertain whether the observed regional volume differences are related to differences in cortical thickness or surface area. Additionally, whether our findings also hold true for youths with OCD remains to be ascertained. Of further note is that data for 530 of the 780 participants included in this study have not been published before; divergent findings here may represent methodological differences, the substantial increase in power in the present study, or the inclusion of additional subjects.

\section{Conclusions}

The results of this study support involvement of the frontal-striatal and (para)limbic circuits and the cerebellum in OCD. Our data indicate that whereas involvement 
of the dorsomedial prefrontal cortex/anterior cingulate cortex/inferior frontal cortex/anterior insula and the cerebellum may be directly related to the pathophysiology of OCD, orbitofrontal-striatal and temporal volume alterations may be secondary to activation-dependent neuroplasticity due to persistent symptoms or compensatory processes related to cognitive dysfunction. This suggests that age is an important contributor to the variability in previous reports on brain structure alterations in OCD. Further insight into the development of structural brain changes in OCD across the lifespan should come from longitudinal studies as well as studies involving pediatric OCD patients.

Received April 29, 2013; revision received Aug. 28, 2013; accepted Sept. 12, 2013 (doi: 10.1176/appi.ajp.2013.13040574). From the Departments of Psychiatry and of Anatomy and Neurosciences, VU University Medical Center, Amsterdam; Department of Psychiatry, Bellvitge University Hospital-Bellvitge Institute for Biomedical Research (IDIBELL), University of Barcelona, Barcelona, Spain; Carlos III Health Institute, Center for Biomedical Research Network on Mental Health (CIBERSAM), Barcelona; Institute of Psychiatry, King's College London, London; MRC Unit on Anxiety and Stress Disorders, Department of Psychiatry, University of Stellenbosch, Stellenbosch, South Africa; Departments of Human Biology and of Psychiatry and Mental Health, University of Cape Town, Cape Town, South Africa; Center of Mathematics, Computation, and Cognition, Federal University of ABC, Santo André, Brazil; Department and Institute of Psychiatry, University of São Paulo Medical School, São Paulo, Brazil; Department of Psychiatry, Academic Medical Center, Amsterdam; Departments of Psychiatry and Radiology, Graduate School of Medical Science, Kyoto Prefectural University of Medicine, Kyoto, Japan; Department of Psychiatry, Seoul National University College of Medicine, Seoul, Republic of Korea; Department of Psychiatry, Western Psychiatric Institute and Clinic, University of Pittsburgh School of Medicine, Pittsburgh; and CRC-Hospital del Mar, Barcelona. Address correspondence to Dr. van den Heuvel (oa.vandenheuvel@ vumc.nl).

The authors report no financial relationships with commercial interests.

Supported by the Dutch Organization for Scientific Research (NWO) (grants 912-02-050, 907-00-012, 940-37-018, and 916.86.038); the Carlos III Health Institute (PI09/01331, PI10/01753, PI10/01003, CP10/00604, and CIBER-CB06/03/0034); the Agency for Administration of University and Research (AGAUR, Barcelona; 2009SGR1554); a "Miguel Servet" contract from the Carlos III Health Institute (CP10/ 00604) to Dr. Soriano-Mas; Ministry of Education, Culture, Sports, Science, and Technology (Japan) Grants-in-Aid for Young Scientists to Dr. Narumoto (23591724) and to Dr. Nakamae (24791223); Wellcome Trust project grant 064846; a grant from the Foundation for the Support of Research in the State of São Paulo (FAPESP) to Dr. Miguel (2005/55628-8); a FAPESP scholarship to Dr. Hoexter (2005/ 04206-6); and a National Research Foundation of Korea grant funded by the Korean government (Ministry of Education, Science, and Technology, 2012-0005150).

\section{References}

1. Menzies L, Chamberlain SR, Laird AR, Thelen SM, Sahakian BJ, Bullmore ET: Integrating evidence from neuroimaging and neuropsychological studies of obsessive-compulsive disorder: the orbitofronto-striatal model revisited. Neurosci Biobehav Rev 2008; 32:525-549

2. Radua J, Mataix-Cols D: Voxel-wise meta-analysis of grey matter changes in obsessive-compulsive disorder. Br J Psychiatry 2009; 195:393-402
3. Radua J, van den Heuvel OA, Surguladze S, Mataix-Cols D: Metaanalytical comparison of voxel-based morphometry studies in obsessive-compulsive disorder vs other anxiety disorders. Arch Gen Psychiatry 2010; 67:701-711

4. Rotge JY, Langbour N, Guehl D, Bioulac B, Jaafari N, Allard M, Aouizerate B, Burbaud P: Gray matter alterations in obsessivecompulsive disorder: an anatomic likelihood estimation metaanalysis. Neuropsychopharmacology 2010; 35:686-691

5. Peng Z, Lui SS, Cheung EF, Jin Z, Miao G, Jing J, Chan RC: Brain structural abnormalities in obsessive-compulsive disorder: converging evidence from white matter and grey matter. Asian J Psychiatr 2012; 5:290-296

6. Tops M, Boksem MA: A potential role of the inferior frontal gyrus and anterior insula in cognitive control, brain rhythms, and event-related potentials. Front Psychol 2011; 2:330

7. de Wit SJ, de Vries FE, van der Werf YD, Cath DC, Heslenfeld DJ, Veltman EM, van Balkom AJ, Veltman DJ, van den Heuvel OA: Presupplementary motor area hyperactivity during response inhibition: a candidate endophenotype of obsessive-compulsive disorder. Am J Psychiatry 2012; 169:1100-1108

8. Pujol J, Soriano-Mas C, Alonso P, Cardoner N, Menchón JM, Deus J, Vallejo J: Mapping structural brain alterations in obsessivecompulsive disorder. Arch Gen Psychiatry 2004; 61:720-730

9. van den Heuvel OA, Remijnse PL, Mataix-Cols $D$, Vrenken $\mathrm{H}$, Groenewegen HJ, Uylings HB, van Balkom AJ, Veltman DJ: The major symptom dimensions of obsessive-compulsive disorder are mediated by partially distinct neural systems. Brain 2009; 132:853-868

10. Togao O, Yoshiura T, Nakao T, Nabeyama M, Sanematsu H, Nakagawa A, Noguchi T, Hiwatashi A, Yamashita K, Nagao E, Kanba S, Honda $\mathrm{H}$ : Regional gray and white matter volume abnormalities in obsessive-compulsive disorder: a voxel-based morphometry study. Psychiatry Res 2010; 184:29-37

11. Riffkin J, Yücel M, Maruff P, Wood SJ, Soulsby B, Olver J, Kyrios M, Velakoulis D, Pantelis C: A manual and automated MRI study of anterior cingulate and orbito-frontal cortices, and caudate nucleus in obsessive-compulsive disorder: comparison with healthy controls and patients with schizophrenia. Psychiatry Res 2005; 138:99-113

12. Hoexter MQ, de Souza Duran FL, D'Alcante CC, Dougherty DD, Shavitt RG, Lopes AC, Diniz JB, Deckersbach T, Batistuzzo MC, Bressan RA, Miguel EC, Busatto GF: Gray matter volumes in obsessive-compulsive disorder before and after fluoxetine or cognitive-behavior therapy: a randomized clinical trial. Neuropsychopharmacology 2012; 37:734-745

13. Tobe RH, Bansal R, Xu D, Hao X, Liu J, Sanchez J, Peterson BS: Cerebellar morphology in Tourette syndrome and obsessivecompulsive disorder. Ann Neurol 2010; 67:479-487

14. Wittfoth M, Bornmann S, Peschel T, Grosskreutz J, Glahn A, Buddensiek N, Becker $H$, Dengler R, Müller-Vahl KR: Lateral frontal cortex volume reduction in Tourette syndrome revealed by VBM. BMC Neurosci 2012; 13:17

15. Salimi-Khorshidi G, Smith SM, Keltner JR, Wager TD, Nichols TE: Meta-analysis of neuroimaging data: a comparison of imagebased and coordinate-based pooling of studies. Neuroimage 2009; 45:810-823

16. van Haren NE, Cahn W, Hulshoff Pol HE, Schnack HG, Caspers E, Lemstra A, Sitskoorn MM, Wiersma D, van den Bosch RJ, Dingemans PM, Schene AH, Kahn RS: Brain volumes as predictor of outcome in recent-onset schizophrenia: a multi-center MRI study. Schizophr Res 2003; 64:41-52

17. Ashburner J: A fast diffeomorphic image registration algorithm. Neuroimage 2007; 38:95-113

18. Ziegler G, Dahnke R, Jäncke L, Yotter RA, May A, Gaser C: Brain structural trajectories over the adult lifespan. Hum Brain Mapp 2012; 33:2377-2389

19. Ridgway GR, Omar R, Ourselin S, Hill DL, Warren JD, Fox NC: Issues with threshold masking in voxel-based morphometry of atrophied brains. Neuroimage 2009; 44:99-111 
20. Kim JJ, Lee MC, Kim J, Kim IY, Kim SI, Han MH, Chang KH, Kwon JS: Grey matter abnormalities in obsessive-compulsive disorder: statistical parametric mapping of segmented magnetic resonance images. Br J Psychiatry 2001; 179:330-334

21. Yoo SY, Roh MS, Choi JS, Kang DH, Ha TH, Lee JM, Kim IY, Kim SI, Kwon JS: Voxel-based morphometry study of gray matter abnormalities in obsessive-compulsive disorder. J Korean Med Sci 2008; 23:24-30

22. Worsley KJ, Marrett S, Neelin P, Vandal AC, Friston KJ, Evans AC: A unified statistical approach for determining significant signals in images of cerebral activation. Hum Brain Mapp 1996; 4:58-73

23. Aoki $\mathrm{Y}$, Aoki A, Suwa $\mathrm{H}$ : Reduction of $\mathrm{N}$-acetylaspartate in the medial prefrontal cortex correlated with symptom severity in obsessive-compulsive disorder: meta-analyses of (1)H-MRS studies. Transcult Psychiatry 2012; 2:e153

24. van Tol MJ, van der Wee NJ, van den Heuvel OA, Nielen MM, Demenescu LR, Aleman A, Renken R, van Buchem MA, Zitman FG, Veltman DJ: Regional brain volume in depression and anxiety disorders. Arch Gen Psychiatry 2010; 67:1002-1011

25. Frodl T, Skokauskas N: Meta-analysis of structural MRI studies in children and adults with attention deficit hyperactivity disorder indicates treatment effects. Acta Psychiatr Scand 2012; 125:114-126

26. Middleton FA, Strick PL: Basal ganglia output and cognition: evidence from anatomical, behavioral, and clinical studies. Brain Cogn 2000; 42:183-200

27. Schutter DJ, van Honk J: The cerebellum on the rise in human emotion. Cerebellum 2005; 4:290-294

28. Keuthen NJ, Makris N, Schlerf JE, Martis B, Savage CR, McMullin K, Seidman LJ, Schmahmann JD, Kennedy DN, Hodge SM, Rauch SL: Evidence for reduced cerebellar volumes in trichotillomania. Biol Psychiatry 2007; 61:374-381

29. Hoppenbrouwers SS, Schutter DJ, Fitzgerald PB, Chen R, Daskalakis ZJ: The role of the cerebellum in the pathophysiology and treatment of neuropsychiatric disorders: a review. Brain Res Brain Res Rev 2008; 59:185-200

30. Johnston MV: Plasticity in the developing brain: implications for rehabilitation. Dev Disabil Res Rev 2009; 15:94-101
31. Kassem MS, Lagopoulos J, Stait-Gardner T, Price WS, Chohan TW, Arnold JC, Hatton SN, Bennett MR: Stress-induced grey matter loss determined by MRI is primarily due to loss of dendrites and their synapses. Mol Neurobiol 2013; 47:645-661

32. Volkow ND, Wang GJ, Fowler JS, Tomasi D, Telang F: Addiction: beyond dopamine reward circuitry. Proc Natl Acad Sci USA 2011; 108:15037-15042

33. Harrison BJ, Soriano-Mas C, Pujol J, Ortiz H, López-Solà M, Hernández-Ribas R, Deus J, Alonso P, Yücel M, Pantelis C, Menchon $\mathrm{JM}$, Cardoner N: Altered corticostriatal functional connectivity in obsessive-compulsive disorder. Arch Gen Psychiatry 2009; 66: 1189-1200

34. Corbetta M, Shulman GL: Control of goal-directed and stimulusdriven attention in the brain. Nat Rev Neurosci 2002; 3:201-215

35. Critchley HD, Wiens S, Rotshtein P, Ohman A, Dolan RJ: Neural systems supporting interoceptive awareness. Nat Neurosci 2004; 7:189-195

36. Calder AJ, Lawrence AD, Young AW: Neuropsychology of fear and loathing. Nat Rev Neurosci 2001; 2:352-363

37. Paulus MP, Stein MB: Interoception in anxiety and depression. Brain Struct Funct 2010; 214:451-463

38. Grieve SM, Clark CR, Williams LM, Peduto AJ, Gordon E: Preservation of limbic and paralimbic structures in aging. Hum Brain Mapp 2005; 25:391-401

39. Sabatinelli D, Fortune EE, Li Q, Siddiqui A, Krafft C, Oliver WT, Beck S, Jeffries J: Emotional perception: meta-analyses of face and natural scene processing. Neuroimage 2011; 54:2524-2533

40. Phelps EA: Human emotion and memory: interactions of the amygdala and hippocampal complex. Curr Opin Neurobiol 2004; 14:198-202

41. Mataix-Cols D, Wooderson S, Lawrence N, Brammer MJ, Speckens A, Phillips ML: Distinct neural correlates of washing, checking, and hoarding symptom dimensions in obsessivecompulsive disorder. Arch Gen Psychiatry 2004; 61:564-576

42. Iervolino AC, Rijsdijk FV, Cherkas L, Fullana MA, Mataix-Cols D: A multivariate twin study of obsessive-compulsive symptom dimensions. Arch Gen Psychiatry 2011; 68:637-644 